\title{
The Topp-Leone Weibull Distribution: Its Properties and Application
}

\author{
Diamond O. Tuoyo, Festus C. Opone* and N. Ekhosuehi \\ Department of Statistics, University of Benin, Nigeria \\ e-mail: festus.opone@physci.uniben.edu*
}

\begin{abstract}
This paper presents a new generalization of the Topp-Leone distribution called the ToppLeone Weibull Distribution (TLWD). Some of the mathematical properties of the proposed distribution are derived, and the maximum likelihood estimation method is adopted in estimating the parameters of the proposed distribution. An application of the proposed distribution alongside with some well-known distributions belonging to the Topp-Leone generated family of distributions, to a real lifetime data set reveals that the proposed distribution exhibits more flexibility in modeling lifetime data based on some comparison criteria such as maximized log-likelihood, Akaike Information Criterion $[A I C=2 k-2 \log (L)], \quad$ Kolmogorov-Smirnov test statistic $(K-S)$ and Anderson Darling test statistic $\left(A^{*}\right)$ and Crammer-Von Mises test statistic $\left(W^{*}\right)$.
\end{abstract}

\section{Introduction}

Lifetime distributions are statistical models used for analyzing real life problems based on survival time. Many statistical distributions have been proposed to model lifetime data and the Topp-Leone distribution introduced by [14] is one of such distributions. In practice, most lifetime data sets encountered exhibits a bathtub hazard rate property and the one parameter Topp-Leone distribution happens to be the simplest distribution with such hazard rate property, but being a single parameter defined on a unit interval, its flexibility is limited in handling lifetime data sets.

Several generalizations of the distribution have been introduced to address the aforementioned drawback. These generalizations are found in the works of [1-5,9,10]. In this paper, we introduce a new generalization of the Topp-Leone distribution which serves as an alternative distribution among the Topp-Leone generated family of

Received: August 5, 2021; Accepted: September 7, 2021

2010 Mathematics Subject Classification: Primary 60E05; Secondary 62E99.

Keywords and phrases: Topp-Leone distribution, Weibull distribution, maximum likelihood estimate. 
distributions. We shall call the proposed distribution, "Topp-Leone Weibull Distribution (TLWD)".

[14] proposed a J-shaped univariate distribution with cumulative distribution function defined by

$$
F(x)=\left(\frac{x}{b}\right)^{\alpha}\left(2-\frac{x}{b}\right)^{\alpha}, \quad 0 \leq x \leq b<\infty, 0<\alpha<1
$$

and the corresponding density function given by

$$
f(x)=\frac{2 \alpha}{b}\left(\frac{x}{b}\right)^{\alpha-1}\left(1-\frac{x}{b}\right)\left(2-\frac{x}{b}\right)^{\alpha-1} .
$$

The survival function and the hazard rate function of Topp-Leone distribution are obtained using equations (1) and (2) as

$$
S(x)=1-F(x)=1-\left(\frac{x}{b}\right)^{\alpha}\left(2-\frac{x}{b}\right)^{\alpha}
$$

and

$$
H(x)=\frac{F(x)}{1-F(x)}=\frac{\frac{2 \alpha}{b}\left(\frac{x}{b}\right)^{\alpha-1}\left(1-\frac{x}{b}\right)}{\left\{\left(2-\frac{x}{b}\right)^{1-\alpha}-\left(\frac{x}{b}\right)^{\alpha}\left(2-\frac{x}{b}\right)\right\}} .
$$

[11] improved on the mathematical properties of the Topp-Leone distribution by deriving higher moments for the distribution and a general usefulness of the distribution. Motivations for generalizing the Topp-Leone distribution arose after the work of [11]. One of such motivation is that, suppose a random variable $x$ follow the Topp-Leone distribution then the random variable $x$ can have either a finite support $(0<x<b)$ or an infinite support $(0<x<b<\infty)$.

Suppose we fix the parameter $b=1$ in equations (1) and (2), then we obtain the cumulative distribution function and the density function of the one parameter ToppLeone distribution defined on a unit interval respectively as

$$
F(x)=x^{\alpha}(2-x)^{\alpha}, \quad 0<x<1, \alpha>0
$$

and

$$
f(x)=2 \alpha x^{\alpha-1}(1-x)(2-x)^{\alpha-1} .
$$

Figure 1 shows the graphical illustration of the density function and the hazard rate function of the Topp-Leone distribution for varying values of the shape parameter defined in the interval $0<\alpha<1$ and a fixed value of the scale parameter $b=1$. 
(a)

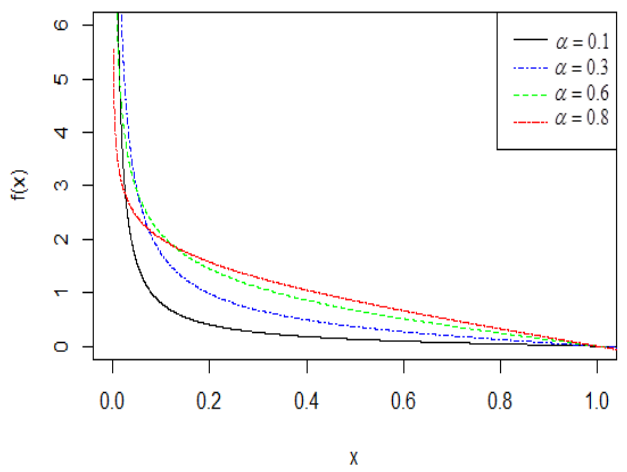

(b)

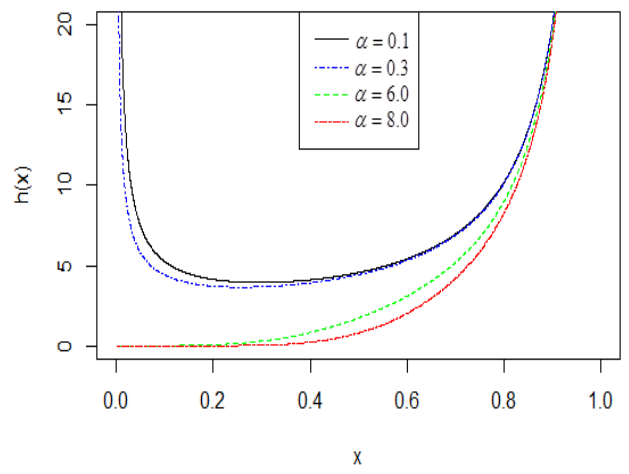

Figure 1: Density and hazard rate functions of the Topp-Leone distribution.

The plots in Figure 1(a), clearly shows that the density function of the TLD exhibits a reversed-J shape for different values of the shape parameter defined in the interval $0<\alpha<1$ and a fixed value of the scale parameter $b=1$, while the plots in Figure 1(b) indicates that the hazard rate function of TLD exhibits a bathtub shape whenever $0<\alpha<1$ and a non-decreasing shape for $\alpha \geq 1$. The remaining sections of this paper are organized as follows: Section 2 presents some mathematical properties of the proposed distribution which include; the probability density function, cumulative distribution function, Hazard rate function, Survival function, Quantile function, Moments, Moment generating function, Renyi entropy, and distribution of ordered statistics. The model parameter estimation and simulation study on the maximum likelihood estimates of the proposed distribution are given in Section 3. Finally, in Section 4, we applied the proposed distribution to a real data set and compared its fit with the fit of some existing Topp-Leone generated family of distributions.

\section{Mathematical Properties of the Proposed Distribution}

\subsection{The density and cumulative distribution functions of the proposed distribution}

[13] introduced a new class of the Topp-Leone generated family of distributions with the cumulative distribution function defined as

$$
F(x)=[G(x)]^{\alpha}[2-G(x)]^{\alpha},
$$

and the corresponding density function given by

$$
f(x)=2 \alpha g(x)(1-G(x))[G(x)]^{\alpha-1}[2-G(x)]^{\alpha-1} .
$$


Taking $G(x)$ as the distribution function of the Exponentiated exponential distribution developed by [8], the authors obtained the cumulative distribution function of the Topp-Leone Generalized Exponential Distribution as

$$
F(x)=\left[1-e^{-\lambda x}\right]^{\beta \alpha}\left[2-\left(1-e^{-\lambda x}\right)^{\beta}\right]^{\alpha},
$$

and the corresponding density function given by

$$
\begin{aligned}
f(x)= & 2 \alpha \beta \lambda e^{-\lambda x}\left[1-e^{-\lambda x}\right]^{\beta \alpha-1}\left(1-\left(1-e^{-\lambda x}\right)^{\beta}\right) \\
& \times\left[2-\left(1-e^{-\lambda x}\right)^{\beta}\right]^{\alpha-1} .
\end{aligned}
$$

Using a similar approach of generating new distributions defined in equations (7) and (8), we assume the random variable $X$ to follow the 2-parameter Weibull distribution with cumulative distribution function and probability density function respectively defined by

$$
G(x)=1-e^{-\theta x^{\alpha}}
$$

and

$$
g(x)=\alpha \theta x^{\alpha-1} e^{-\theta x^{\alpha}} .
$$

Inserting equations (11) and (12) into (7) and (8), we define the cumulative distribution function and the probability distribution function of the Topp Leone Weibull distribution (TLWD) respectively as

$$
\begin{aligned}
F(x) & =\left\{\left(1-e^{-\theta x^{\alpha}}\right)\left[\left(2-\left(1-e^{-\theta x^{\alpha}}\right)\right]\right\}^{\lambda}\right. \\
& =\left\{1-e^{-2 \theta x^{\alpha}}\right\}^{\lambda}
\end{aligned}
$$

and

$$
\begin{aligned}
f(x) & =2 \lambda \alpha \theta x^{\alpha-1} e^{-\theta x^{\alpha}}\left[1-\left(1-e^{-\theta x^{\alpha}}\right)\right]\left[\left(1-e^{-\theta x^{\alpha}}\right)\right]^{\lambda-1}\left[2-\left(1-e^{-\theta x^{\alpha}}\right)\right]^{\lambda-1} \\
& =2 \lambda \alpha \theta x^{\alpha-1} e^{-2 \theta x^{\alpha}}\left(1-e^{-2 \theta x^{\alpha}}\right)^{\lambda-1}
\end{aligned}
$$

The series representation of equation (14) can be obtain as

$$
\begin{aligned}
& \left(1-e^{-2 \theta x^{\alpha}}\right)^{\lambda-1}=\sum_{j=0}^{\infty}\left(\begin{array}{c}
\lambda-1 \\
j
\end{array}\right)(-1)^{j} e^{-2 \theta x^{\alpha} j} \\
& f(x)=2 \lambda \alpha \theta \sum_{j=0}^{\infty}\left(\begin{array}{c}
\lambda-1 \\
j
\end{array}\right)(-1)^{j} x^{\alpha-1} e^{-2 \theta x^{\alpha}(1+j) .}
\end{aligned}
$$


The graphical plots of the probability density function are shown in Figure 2.

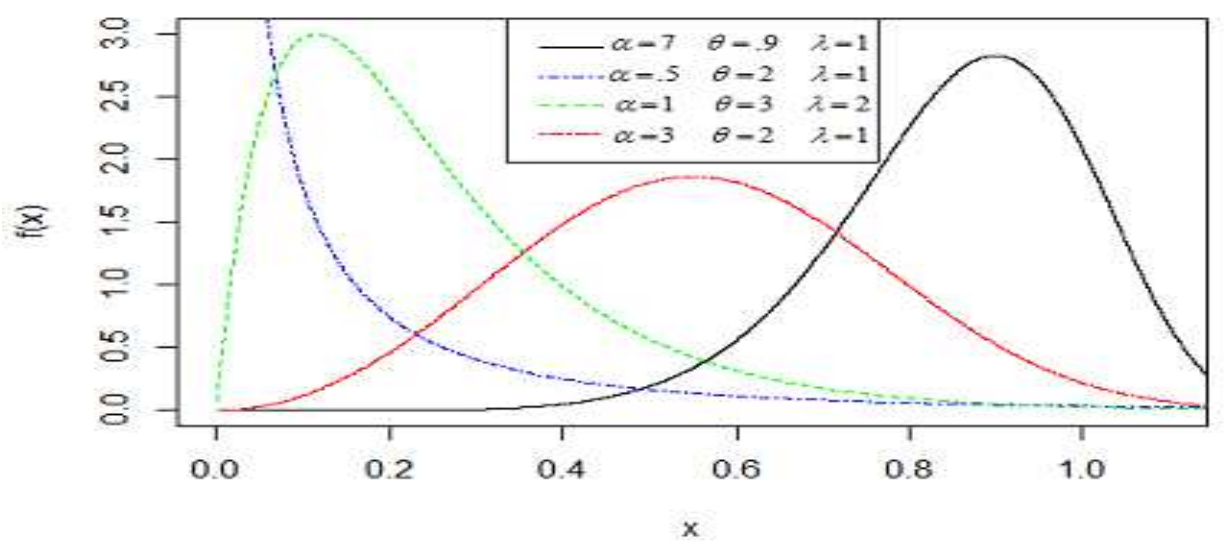

Figure 2: Density function of the TLWD for fixed value of the parameters.

The plots in Figure 2 clearly indicate that the density function of the TLWD exhibits a left-skewed, right-skewed, reversed J-shape and symmetric unimodal shapes.

\subsection{Survival function and hazard function of the proposed distribution}

Using equations (13) and (14), the survival function and the hazard rate function of the Topp Leone Weibull distribution are given by

$$
\begin{aligned}
S(x) & =1-F(x) \\
& =1-\left\{1-e^{-2 \theta x^{\alpha}}\right\}^{\lambda}
\end{aligned}
$$

and

$$
\begin{aligned}
H(x) & =\frac{f(x)}{1-F(x)} \\
& =\frac{2 \lambda \alpha \theta x^{\alpha-1} e^{-2 \theta x^{\alpha}}}{\left\{\left(1-e^{-2 \theta x^{\alpha}}\right)^{1-\lambda}-\left(1-e^{-2 \theta x^{\alpha}}\right)\right\}}
\end{aligned}
$$

The graphical plots of the hazard rate function of the TLWD for different choice of the parameter value is shown in Figure 3. 


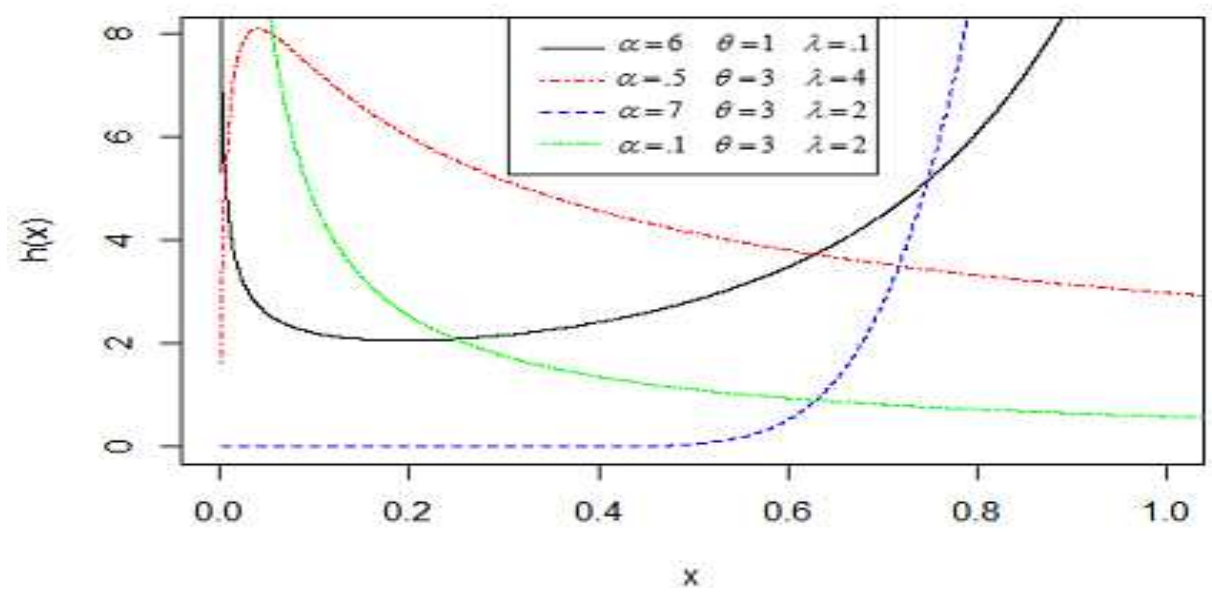

Figure 3: Hazard rate function of the TLWD for fixed value of the parameters.

\subsection{Quantile function of the proposed distribution}

The quantile function of a random variable $x$ is obtained by solving the system equation of $F(x)=p$. Thus, given the cumulative distribution function $F(x)$ defined in equation (13), the $p^{\text {th }}$ quantile function of the TLWD can be obtain as

$$
\begin{aligned}
& \left\{1-e^{-2 \theta x^{\alpha}}\right\}^{\lambda}=p \\
& 1-e^{-2 \theta x^{\alpha}}=p^{1 / \lambda} \\
& e^{-2 \theta x^{\alpha}}=1-p^{1 / \lambda}
\end{aligned}
$$

taking the natural logarithm of both sides we have

$$
\begin{aligned}
& -2 \theta x^{\alpha}=\log \left(1-p^{1 / \lambda}\right) \\
& x^{\alpha}=-\frac{1}{2 \theta} \log \left(1-p^{1 / \lambda}\right) \\
& x=\left\{-\frac{1}{2 \theta} \log \left(1-p^{1 / \lambda}\right)\right\}^{1 / \alpha}, \quad 0<p<1 .
\end{aligned}
$$

The median of the TLWD can be obtained by substituting $p=1 / 2$ in equation (18) which yields,

$$
\text { median }=\left\{-\frac{1}{2 \theta} \log \left(1-(0.5)^{1 / \lambda}\right)\right\}^{1 / \alpha}
$$




\subsection{Moments of the proposed distribution}

Let $X$ be a continuous random variable with density function $f(x)$, then the $r^{\text {th }}$ moment about the origin of $X$ is defined by

$$
E\left[X^{r}\right]=\int_{-\infty}^{\infty} x^{r} f(x) d x
$$

inserting the density function of the TLWD into equation (20), the $r^{\text {th }}$ moment about the origin of the random variable $X$ is defined by

$$
\begin{gathered}
E\left[X^{r}\right]=\int_{0}^{\infty} 2 \lambda \alpha \theta x^{r+\alpha-1} e^{-2 \theta x^{\alpha}}\left\{1-e^{-2 \theta x^{\alpha}}\right\}^{\lambda-1} d x \\
\left\{1-e^{-2 \theta x^{\alpha}}\right\}^{\lambda-1}=\sum_{j=0}^{\infty}\left(\begin{array}{c}
\lambda-1 \\
j
\end{array}\right)(-1)^{j} e^{-2 \theta x^{\alpha} j}
\end{gathered}
$$

so that equation (21) now becomes,

$$
E\left[X^{r}\right]=2 \lambda \alpha \theta \sum_{j=0}^{\infty}\left(\begin{array}{c}
\lambda-1 \\
j
\end{array}\right)(-1)^{j} \int_{0}^{\infty} x^{r+\alpha-1} e^{-2 \theta x^{\alpha}(1+j)} d x
$$

evaluating the integral part of equation (22), we have

$$
\int_{0}^{\infty} x^{r+\alpha-1} e^{-2 \theta x^{\alpha}(1+j)} d x
$$

let $y=2 \theta x^{\alpha}(1+j)$, which implies that, $x=\left[\frac{y}{2 \theta(1+j)}\right]^{1 / \alpha}$

so that, $d x=\frac{1}{\alpha 2 \theta(1+j)}\left[\frac{y}{2 \theta(1+j)}\right]^{1 / \alpha-1} d y$

$$
\begin{aligned}
& \int_{0}^{\infty}\left\{\left[\frac{y}{2 \theta(1+j)}\right]^{1 / \alpha}\right\}^{r+\alpha-1} e^{-y} \frac{1}{\alpha 2 \theta(1+j)}\left[\frac{y}{2 \theta(1+j)}\right]^{1 / \alpha-1} d y \\
& \int_{0}^{\infty} \frac{y^{r / \alpha^{+1-1}-\alpha^{+1 / \alpha-1}}}{\alpha[2 \theta(1+j)]^{r / \alpha^{+1-1 / \alpha}+\alpha^{+1 / \alpha}}} e^{-y} d y \\
& \int_{0}^{\infty} \frac{y^{r / \alpha} e^{-y}}{\alpha[2 \theta(1+j)]^{r / \alpha+1}} d y=\frac{\Gamma\left(r / \alpha^{+1}\right)}{\alpha[2 \theta(1+j)]^{r / \alpha+1}} .
\end{aligned}
$$

Thus, the $r^{t h}$ moment about the origin of the TLWD is given by

$$
E\left[X^{r}\right]=2 \lambda \theta \sum_{j=0}^{\infty}\left(\begin{array}{c}
\lambda-1 \\
j
\end{array}\right)(-1)^{j} \frac{\Gamma\left(r / \alpha^{+1}\right)}{[2 \theta(1+j)]^{r / \alpha+1}} .
$$

The first four raw moments of the TLWD are obtained from equation (23) as 


$$
\begin{aligned}
& \mu_{1}^{\prime}=2 \lambda \theta \sum_{j=0}^{\infty}\left(\begin{array}{c}
\lambda-1 \\
j
\end{array}\right)(-1)^{j} \frac{\Gamma(1 / \alpha+1)}{[2 \theta(1+j)]^{1 / \alpha+1}}, \\
& \mu_{2}^{\prime}=2 \lambda \theta \sum_{j=0}^{\infty}\left(\begin{array}{c}
\lambda-1 \\
j
\end{array}\right)(-1)^{j} \frac{\Gamma(2 / \alpha+1)}{[2 \theta(1+j)]^{2 / \alpha+1}}, \\
& \mu_{3}^{\prime}=2 \lambda \theta \sum_{j=0}^{\infty}\left(\begin{array}{c}
\lambda-1 \\
j
\end{array}\right)(-1)^{j} \frac{\Gamma\left(3 / \alpha^{+1}\right)}{[2 \theta(1+j)]^{3 / \alpha+1}}, \\
& \mu_{4}^{\prime}=2 \lambda \theta \sum_{j=0}^{\infty}\left(\begin{array}{c}
\lambda-1 \\
j
\end{array}\right)(-1)^{j} \frac{\Gamma(4 / \alpha+1)}{[2 \theta(1+j)]^{4 / \alpha+1}} .
\end{aligned}
$$

Furthermore, using the first four raw moments defined above, the measures of skewness $\left(S_{k}\right)$ and kurtosis $\left(K_{S}\right)$ are obtained as

$$
S_{k}=\frac{\mu_{3}^{\prime}-3 \mu_{2}^{\prime} \mu+2 \mu^{3}}{\left(\mu_{2}^{\prime}-\mu^{2}\right)^{3 / 2}} \quad \text { and } \quad K_{S}=\frac{\mu_{4}^{\prime}-4 \mu_{3}^{\prime} \mu+6 \mu_{2}^{\prime} \mu^{2}-3 \mu^{4}}{\left(\mu_{2}^{\prime}-\mu^{2}\right)^{2}} .
$$

Tables 1 and 2 show the theoretical moments of the TLWD for different values of the parameters.

Table 1: Theoretical moments of TLWD for fixed value of the parameter $(\alpha=2)$.

\begin{tabular}{ccccc}
\hline$\mu_{r}^{\prime}$ & $(\theta=2, \lambda=3)$ & $(\theta=2, \lambda=5)$ & $(\theta=4, \lambda=3)$ & $(\theta=4, \lambda=5)$ \\
\hline$\mu_{1}^{\prime}$ & 0.6452 & 0.7310 & 0.4562 & 0.5169 \\
$\mu_{2}^{\prime}$ & 0.4583 & 0.5708 & 0.2292 & 0.2854 \\
$\mu_{3}^{\prime}$ & 0.3542 & 0.4741 & 0.1252 & 0.1676 \\
$\mu_{4}^{\prime}$ & 0.2951 & 0.4173 & 0.0738 & 0.1043 \\
$\sigma^{2}$ & 0.0420 & 0.0364 & 0.0211 & 0.0182 \\
$S_{k}$ & 0.4980 & 0.5143 & 0.4581 & 0.5078 \\
$K_{s}$ & 3.2886 & 3.3859 & 3.5931 & 3.4248 \\
\hline
\end{tabular}

Table 2: Theoretical moments of TLWD for fixed value of the parameter $(\alpha=5)$.

\begin{tabular}{ccccc}
\hline$\mu_{r}^{\prime}$ & $(\theta=2, \lambda=3)$ & $(\theta=2, \lambda=5)$ & $(\theta=4, \lambda=3)$ & $(\theta=4, \lambda=5)$ \\
\hline$\mu_{1}^{\prime}$ & 0.8288 & 0.8750 & 0.7215 & 0.7617 \\
$\mu_{2}^{\prime}$ & 0.6986 & 0.7740 & 0.5294 & 0.5866 \\
$\mu_{3}^{\prime}$ & 0.5982 & 0.6921 & 0.3946 & 0.4566 \\
$\mu_{4}^{\prime}$ & 0.5199 & 0.6254 & 0.2986 & 0.3592 \\
$\sigma^{2}$ & 0.0117 & 0.0084 & 0.0088 & 0.0064 \\
$S_{k}$ & -0.1406 & 0.2517 & -0.1393 & 0.0333 \\
$K_{s}$ & 3.3840 & 0.9577 & 4.4313 & 5.0395 \\
\hline
\end{tabular}


From Tables 1 and 2, we observed that the TLWD can be right skewed $\left(S_{k}>0\right)$, left skewed $\left(S_{k}<0\right)$ and approximately symmetric $\left(S_{k} \approx 0\right)$. Also, at some fixed values of the parameters, the distribution can be leptokurtic $\left(K_{S}>3\right)$, platykurtic $\left(K_{S}<3\right)$ as well as mesokurtic $\left(K_{S} \approx 3\right)$. This claim was clearly shown in Figure 2 .

\subsection{Moment generating function of the proposed distribution}

Let $X$ be a continuous random variable with density function $f(x)$, then the moment generating function of $X$ is defined by

$$
M_{X}(t)=\int_{-\infty}^{\infty} e^{t x} f(x) d x
$$

inserting the density function of the TLWD into equation (24), we obtain the moment generating function of the TLWD as

$$
M_{X}(t)=\int_{0}^{\infty} e^{t x} 2 \lambda \alpha \theta x^{\alpha-1} e^{-2 \theta x^{\alpha}}\left\{1-e^{-2 \theta x^{\alpha}}\right\}^{\lambda-1} d x
$$

using the series representation,

$$
\begin{aligned}
\left\{1-e^{-2 \theta x^{\alpha}}\right\}^{\lambda-1} & =\sum_{j=0}^{\infty}\left(\begin{array}{c}
\lambda-1 \\
j
\end{array}\right)(-1)^{j} e^{-2 \theta x^{\alpha} j} \\
e^{t x} & =\sum_{m=0}^{\infty} \frac{(t x)^{m}}{m !}
\end{aligned}
$$

equation (25) becomes,

$$
M_{X}(t)=2 \lambda \alpha \theta \sum_{j=0}^{\infty} \sum_{M=0}^{\infty}\left(\begin{array}{c}
\lambda-1 \\
j
\end{array}\right)(-1)^{j} \frac{t^{m}}{m !} \int_{0}^{\infty} x^{m+\alpha-1} e^{-2 \theta x^{\alpha}(1+j)} d x
$$

evaluating the integral part of equation (26), we have

$$
\begin{aligned}
& \int_{0}^{\infty} x^{m+\alpha-1} e^{-2 \theta x^{\alpha}(1+j)} d x \\
& =\int_{0}^{\infty}\left\{\left[\frac{y}{2 \theta(1+j)}\right]^{1 / \alpha}\right\}^{m+\alpha-1} e^{-y} \frac{1}{\alpha 2 \theta(1+j)}\left[\frac{y}{2 \theta(1+j)}\right]^{1 / \alpha-1} d y
\end{aligned}
$$

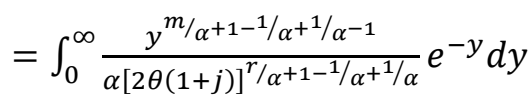

$$
\begin{aligned}
& =\int_{0}^{\infty} \frac{y^{m / \alpha} e^{-y}}{\alpha[2 \theta(1+j)]^{m / \alpha^{+1}}} d y \\
& \int_{0}^{\infty} x^{m+\alpha-1} e^{-2 \theta x^{\alpha}(1+j)} d x=\frac{\Gamma\left(m / \alpha^{+1}\right)}{\alpha[2 \theta(1+j)]^{m / \alpha+1}}
\end{aligned}
$$

upon substituting equation (27) into equation (26), the moment generating function of the 
TLWD is given by

$$
M_{X}(t)=2 \lambda \theta \sum_{j=0}^{\infty} \sum_{m=0}^{\infty}\left(\begin{array}{c}
\lambda-1 \\
j
\end{array}\right)(-1)^{j} \frac{t^{m}}{m !} \frac{\Gamma\left(m / \alpha^{+1}\right)}{[2 \theta(1+j)]^{m / \alpha+1}}
$$

\subsection{Renyi entropy of the proposed distribution}

[12] defined an entropy of a random variable $X$ as a measure of variation of uncertainty associated with the random variable $X$. The Renyi entropy of $X$ with density function $f(x)$, is defined by,

$$
\tau_{R}(\gamma)=\frac{1}{1-\gamma} \log \int f^{\gamma}(x) d x, \quad \gamma>0, \quad \gamma \neq 1
$$

inserting the density function of the TLWD into equation (29), we obtain the Renyi entropy of the random variable $X$ following the TLWD as

$$
\tau_{R}(\gamma)=\frac{1}{1-\gamma} \log \left[(2 \lambda \alpha \theta)^{\gamma} \int_{0}^{\infty} x^{(\alpha-1) \gamma} e^{-2 \theta x^{\alpha} \gamma}\left\{1-e^{-2 \theta x^{\alpha}}\right\}^{(\lambda-1) \gamma} d x\right]
$$

using series representation,

$$
\left\{1-e^{-2 \theta x^{\alpha}}\right\}^{\lambda \gamma-\gamma}=\sum_{j=0}^{\infty}\left(\begin{array}{c}
\lambda \gamma-\gamma \\
j
\end{array}\right)(-1)^{j} e^{-2 \theta x^{\alpha} j}
$$

substituting equation (31) into equation (30), we have

$$
=\frac{1}{1-\gamma} \log \left[(2 \lambda \alpha \theta)^{\gamma} \sum_{j=0}^{\infty}\left(\begin{array}{c}
\lambda \gamma-\gamma \\
j
\end{array}\right)(-1)^{j} \int_{0}^{\infty} x^{(\alpha-1) \gamma} e^{-2 \theta x^{\alpha}(\gamma+j)} d x\right]
$$

again, evaluating the integral part of equation (32),

$$
\begin{aligned}
& \int_{0}^{\infty} x^{(\alpha-1) \gamma} e^{-2 \theta x^{\alpha}(\gamma+j)} d x \\
= & \int_{0}^{\infty}\left\{\left[\frac{y}{2 \theta(\gamma+j)}\right]^{1 / \alpha}\right\}^{\alpha \gamma-\gamma} e^{-y} \frac{1}{\alpha 2 \theta(\gamma+j)}\left[\frac{y}{2 \theta(\gamma+j)}\right]^{1 / \alpha-1} d y \\
= & \int_{0}^{\infty} \frac{y^{\gamma-\gamma / \alpha+1 / \alpha-1}}{\alpha[2 \theta(\gamma+j)]^{\gamma-\gamma / \alpha+1 / \alpha}} e^{-y} d y \\
= & \int_{0}^{\infty} \frac{y^{\gamma+}{ }^{(1-\gamma) / \alpha-1} e^{-y}}{\alpha[2 \theta(1+j)]^{\gamma+{ }^{(1-\gamma) / \alpha}}} d y \\
& \int_{0}^{\infty} x^{(\alpha-1) \gamma} e^{-2 \theta x^{\alpha}(\gamma+j)} d x=\frac{\Gamma\left(\gamma+{ }^{(1-\gamma) / \alpha}\right)}{\alpha[2 \theta(\gamma+j)]^{\gamma+(1-\gamma) / \alpha}}
\end{aligned}
$$

upon substituting equation (33) into equation (32), the Renyi entropy of the random 
variable $X$ following the TLWD is given by

$$
\tau_{R}(\gamma)=\frac{1}{1-\gamma} \log \left[(2 \lambda \alpha \theta)^{\gamma} \sum_{j=0}^{\infty}\left(\begin{array}{c}
\lambda \gamma-\gamma \\
j
\end{array}\right)(-1)^{j} \frac{\Gamma\left(\gamma+{ }^{(1-\gamma)} / \alpha\right)}{\alpha[2 \theta(\gamma+j)]^{\gamma+(1-\gamma) / \alpha}}\right] .
$$

\subsection{The distribution of the ordered statistics of the proposed distribution}

Suppose that $Y_{1: n}<Y_{2: n}<\cdots<Y_{n: n}$ is the order statistics of a random sample generated from TLWD, then the probability density function of the $k^{\text {th }}$ order statistics, say $X=Y_{n: n}$ is given by

$$
h_{i}(x)=\frac{n !}{(n-k) !(k-1) !}[F(x)]^{k-1}[1-F(x)]^{n-k} f(x)
$$

substituting the cumulative distribution function and the density function of TLWD defined in equations (13) and (14), into equation (35), we have

$$
\begin{aligned}
h_{i}(x)= & \frac{2 \lambda \alpha \theta n !}{(n-k) !(k-1) !} \sum_{i=0}^{\infty} \sum_{j=0}^{\infty}\left(\begin{array}{c}
n-k \\
i
\end{array}\right)\left(\begin{array}{c}
\lambda-1 \\
j
\end{array}\right)(-1)^{i+j} e^{-2 \theta x^{\alpha}(1+j)} \\
& \times x^{\alpha-1}\left[\left\{1-e^{-2 \theta x^{\alpha}}\right\}^{\lambda}\right]^{i+k-1}
\end{aligned}
$$

using the series representation,

$$
\left\{1-e^{-2 \theta x^{\alpha}}\right\}^{\lambda(i+k-1)}=\sum_{m=0}^{\infty}\left(\begin{array}{c}
\lambda(i+k-1) \\
m
\end{array}\right)(-1)^{m} e^{-2 \theta x^{\alpha} m}
$$

so that equation (36) becomes,

$$
\begin{aligned}
h_{i}(x)= & \frac{2 \lambda \alpha \theta n !}{(n-k) !(k-1) !} \sum_{i=0}^{\infty} \sum_{j=0}^{\infty} \sum_{j=0}^{\infty}\left(\begin{array}{c}
n-k \\
i
\end{array}\right)\left(\begin{array}{c}
\lambda-1 \\
j
\end{array}\right)\left(\begin{array}{c}
\lambda(i+k-1) \\
m
\end{array}\right) \\
& \times(-1)^{i+j+m} x^{\alpha-1} e^{-2 \theta x^{\alpha}(1+j+m)}
\end{aligned}
$$

Hence, the $s^{t h}$ moment of the $k^{t h}$ order statistics from the TLWD is defined by

$$
\begin{aligned}
E\left(X_{k}^{S}\right)= & \int_{0}^{\infty} x^{s} h_{i}(x) d x \\
= & \frac{2 \lambda \alpha \theta n !}{(n-k) !(k-1) !} \sum_{i=0}^{\infty} \sum_{j=0}^{\infty} \sum_{j=0}^{\infty}\left(\begin{array}{c}
n-k \\
i
\end{array}\right)\left(\begin{array}{c}
\lambda-1 \\
j
\end{array}\right)\left(\begin{array}{c}
\lambda(i+k-1) \\
m
\end{array}\right) \\
& \times(-1)^{i+j+m} \int_{0}^{\infty} x^{S+\alpha-1} e^{-2 \theta x^{\alpha}(1+j+m)}
\end{aligned}
$$

evaluating the integral part of (39),

$$
\int_{0}^{\infty} x^{s+\alpha-1} e^{-2 \theta x^{\alpha}(1+j+m)}
$$


let $y=2 \theta x^{\alpha}(1+j+m)$, which implies that, $x=\left[\frac{y}{2 \theta(1+j+m)}\right]^{1 / \alpha}$

so that, $\quad d x=\frac{1}{\alpha 2 \theta(1+j+m)}\left[\frac{y}{2 \theta(1+j+m)}\right]^{1 / \alpha-1} d y$

$$
\begin{aligned}
& \int_{0}^{\infty}\left\{\left[\frac{y}{2 \theta(1+j+m)}\right]^{1 / \alpha}\right\}^{s+\alpha-1} e^{-y} \frac{1}{\alpha 2 \theta(1+j+m)}\left[\frac{y}{2 \theta(1+j+m)}\right]^{1 / \alpha-1} d y \\
& \int_{0}^{\infty} \frac{y^{S / \alpha+1-1 / \alpha^{+1 / \alpha^{-1}}}}{\alpha[2 \theta(1+j+m)]^{S / \alpha^{+1-1 / \alpha}+\alpha^{1 / \alpha}}} e^{-y} d y \\
& \int_{0}^{\infty} \frac{y^{s / \alpha} e^{-y}}{\alpha[2 \theta(1+j+m)]^{s / \alpha+1}} d y=\frac{\Gamma\left(s / \alpha^{+1}\right)}{\alpha[2 \theta(1+j+m)]^{s / \alpha+1}}
\end{aligned}
$$

equation (39) now becomes

$$
\begin{gathered}
E\left(X_{k}^{S}\right)=\frac{2 \lambda \theta n !}{(n-k) !(k-1) !} \sum_{i=0}^{\infty} \sum_{j=0}^{\infty} \sum_{j=0}^{\infty}\left(\begin{array}{c}
n-k \\
i
\end{array}\right)\left(\begin{array}{c}
\lambda-1 \\
j
\end{array}\right)\left(\begin{array}{c}
\lambda(i+k-1) \\
m
\end{array}\right) \\
\times(-1)^{i+j+m} \frac{\Gamma(s / \alpha+1)}{[2 \theta(1+j+m)]^{S / \alpha+1}} .
\end{gathered}
$$

\subsection{Parameter Estimation of the Proposed Distribution}

\subsection{Maximum likelihood estimation}

Let $\left(x_{1}, x_{2}, \ldots, x_{n}\right)$ be random samples from the TLWD, then the log-likelihood function of the TLWD is defined by

$$
\begin{aligned}
\ell(x, \phi)= & \sum_{i=1}^{n} \log \left[2 \lambda \alpha \theta x^{\alpha-1} e^{-2 \theta x^{\alpha}}\left\{1-e^{-2 \theta x^{\alpha}}\right\}^{\lambda-1}\right], \quad \phi=(\alpha, \theta, \lambda)^{T} \\
= & n \log (2 \lambda \alpha \theta)+(\alpha-1) \sum_{i=1}^{n} \log \left(x_{i}\right)-2 \theta \sum_{i=1}^{n} x_{i}^{\alpha} \\
& +(\lambda-1) \sum_{i=1}^{n} \log \left(1-e^{-2 \theta x^{\alpha}}\right) .
\end{aligned}
$$

On differentiating the log-likelihood function with respect to the parameters, we obtain the score function as,

$$
\begin{aligned}
& \frac{\partial \ell}{\partial \alpha}=\frac{n}{\alpha}+\sum_{i=1}^{n} \log \left(x_{i}\right)-2 \theta \sum_{i=1}^{n} x_{i}^{\alpha} \log \left(x_{i}\right)+2 \theta(\lambda-1) \sum_{i=1}^{n} \frac{x_{i}^{\alpha} \log \left(x_{i}\right) e^{-2 \theta x_{i}^{\alpha}}}{\left\{1-e^{-2 \theta x_{i}^{\alpha}}\right\}} \\
& \frac{\partial \ell}{\partial \lambda}=\frac{n}{\lambda}+\sum_{i=1}^{n} \log \left(1-e^{-2 \theta x_{i}^{\alpha}}\right)
\end{aligned}
$$




$$
\frac{\partial \ell}{\partial \theta}=\frac{n}{\theta}-2 \sum_{i=1}^{n} x_{i}^{\alpha}+2(\lambda-1) \sum_{i=1}^{n} \frac{x_{i}^{\alpha} e^{-2 \theta x_{i}^{\alpha}}}{\left\{1-e^{-2 \theta x_{i}^{\alpha}}\right\}} .
$$

The maximum likelihood estimator $\hat{\phi}$ of $\phi$ can be obtained by solving the system of equation $\frac{\partial \ell}{\partial \phi}=0$. This equation cannot be solved using direct method since the system of equation is non-linear. However, an iterative method of solution such as the Newton Raphson method can be used. The Newton Raphson iterative scheme has the form:

$$
\hat{\phi}=\phi_{k}-H^{-1}\left(\phi_{k}\right) U\left(\phi_{k}\right), \phi=(\alpha, \theta, \lambda)^{T}
$$

where $U\left(\phi_{k}\right)$ is the score function and $H\left(\phi_{k}\right)$ is the Hessian matrix, which is the second derivative of the log-likelihood function. The Hessian matrix is defined by

$$
H\left(\phi_{k}\right)=\left[\begin{array}{ccc}
\frac{\partial^{2} \ell}{\partial \alpha \partial \alpha} & \frac{\partial^{2} \ell}{\partial \alpha \partial \theta} & \frac{\partial^{2} \ell}{\partial \alpha \partial \lambda} \\
\frac{\partial^{2} \ell}{\partial \theta \partial \alpha} & \frac{\partial^{2} \ell}{\partial \theta \partial \theta} & \frac{\partial^{2} \ell}{\partial \theta \partial \lambda} \\
\frac{\partial^{2} \ell}{\partial \lambda \partial \alpha} & \frac{\partial^{2} \ell}{\partial \lambda \partial \theta} & \frac{\partial^{2} \ell}{\partial \lambda \partial \lambda}
\end{array}\right]
$$

where,

$$
\begin{aligned}
& \frac{\partial^{2} \ell}{\partial \alpha \partial \alpha}=-\frac{n}{\alpha^{2}}-2 \theta \sum_{i=1}^{n} x_{i}^{\alpha}\left[\log \left(x_{i}\right)\right]^{2}+2 \theta(\lambda-1) \sum_{i=1}^{n} \frac{x_{i}^{\alpha}\left[\log \left(x_{i}\right)\right]^{2} e^{-2 \theta x_{i}^{\alpha}}\left(1-2 \theta x_{i}^{\alpha}-e^{-2 \theta x_{i}^{\alpha}}\right)}{\left\{1-e^{-2 \theta x_{i}^{\alpha}}\right\}^{2}} \\
& \frac{\partial^{2} \ell}{\partial \alpha \partial \theta}=\frac{\partial^{2} \ell}{\partial \theta \partial \alpha}=-2 \sum_{i=1}^{n} x_{i}^{\alpha} \log \left(x_{i}\right)+2(\lambda-1) \sum_{i=1}^{n} \frac{x_{i}^{\alpha} \log \left(x_{i}\right) e^{-2 \theta x_{i}^{\alpha}}\left(1-2 \theta x_{i}^{\alpha}-e^{-2 \theta x_{i}^{\alpha}}\right)}{\left\{1-e^{-2 \theta x_{i}^{\alpha}}\right\}^{2}} \\
& \frac{\partial^{2} \ell}{\partial \theta \partial \lambda}=\frac{\partial^{2} \ell}{\partial \lambda \partial \theta}=2 \sum_{i=1}^{n} \frac{x_{i}^{\alpha} e^{-2 \theta x_{i}^{\alpha}}}{\left\{1-e^{-2 \theta x_{i}^{\alpha}}\right\}} \\
& \frac{\partial^{2} \ell}{\partial \alpha \partial \lambda}=\frac{\partial^{2} \ell}{\partial \lambda \partial \alpha}=2 \theta \sum_{i=1}^{n} \frac{x_{i}^{\alpha} \log \left(x_{i}\right) e^{-2 \theta x_{i}^{\alpha}}}{\left\{1-e^{-2 \theta x_{i}^{\alpha}}\right\}} \\
& \frac{\partial^{2} \ell}{\partial \lambda \partial \lambda}=-\frac{n}{\lambda^{2}} \\
& \frac{\partial^{2} \ell}{\partial \theta \partial \theta}=-\frac{n}{\theta^{2}}-4(\lambda-1) \sum_{i=1}^{n} \frac{x_{i}^{2 \alpha} e^{-2 \theta x_{i}^{\alpha}}}{\left\{1-e^{-2 \theta x_{i}^{\alpha}}\right\}^{2}}
\end{aligned}
$$

\subsection{Interval estimate}

The asymptotic confidence intervals (CIs) for the parameters of TLWD $(\alpha, \theta, \lambda)$ are 
obtained according to the asymptotic distribution of the maximum likelihood estimates of the parameters.

Suppose $\hat{\phi}=(\hat{\alpha}, \hat{\theta}, \hat{\lambda})$ is MLE of $\phi$, then the estimators are approximately bi-variate normal with mean $(\alpha, \theta, \lambda)$ and the Fisher information matrix is given by:

$$
I\left(\phi_{k}\right)=-E\left(H\left(\phi_{k}\right)\right) .
$$

The approximate $(1-\delta) 100 \mathrm{CIs}$ for the parameters $\alpha, \theta$ and $\lambda$ respectively, are

$$
\hat{\alpha} \pm Z_{\delta / 2} \sqrt{\operatorname{var}(\hat{\alpha})}, \hat{\theta} \pm Z_{\delta / 2} \sqrt{\operatorname{var}(\hat{\theta})} \text { and } \hat{\lambda} \pm Z_{\delta / 2} \sqrt{\operatorname{var}(\hat{\lambda})}
$$

where $\operatorname{var}(\hat{\alpha}), \operatorname{var}(\hat{\theta})$ and $\operatorname{var}(\hat{\lambda})$ are the variance of $\alpha, \theta$ and $\lambda$ which are given by the diagonal elements of the variance-covariance matrix $I^{-1}\left(\phi_{k}\right)$ and $Z_{\delta / 2}$ is the upper $(\delta / 2)$ percentile of the standard normal distribution.

\subsection{Simulation Study}

In this section, we investigate the asymptotic behaviour of the maximum likelihood estimates of the parameters of the Topp-Leone Weibull distribution (TLWD) through a simulation study. A Monte Carlo simulation study is repeated 1000 times for different sample sizes $n=50,75,100,200$ and parameter values $(\alpha=1.0, \theta=0.3, \lambda=0.5)$, $(\alpha=1.0, \theta=0.3, \lambda=0.5)$ and $(\alpha=1.0, \theta=0.3, \lambda=0.5)$. [6] suggested an algorithm for the simulation study employing the following steps.

1. Choose the value $N$ (i.e. number of Monte Carlo simulation);

2. choose the values $\phi_{0}=\left(\alpha_{0}, \theta_{0}, \lambda_{0}\right)$ corresponding to the parameters of the $\operatorname{TLWD}(\alpha, \theta, \lambda)$;

3. generate a sample of size $n$ from TLWD;

4. compute the maximum likelihood estimates $\hat{\phi}_{0}$ of $\phi_{0}$;

5. repeat steps (3-4), $N$-times;

6. compute the:

(i) Average Bias $=\frac{1}{N} \sum_{i=1}^{N}\left(\widehat{\phi}_{\iota}-\phi_{0}\right)$,

(ii) Mean Square Error (MSE) $=\frac{1}{N} \sum_{i=1}^{N}\left(\widehat{\phi}_{l}-\phi_{0}\right)^{2}$ and 
(iii) Coverage Probability of the $95 \%$ confidence interval of the estimates $\hat{\phi}_{i}$ given by

$$
C P\left(\phi_{0}\right)=\frac{1}{N} \sum_{i=1}^{N} I\left(\hat{\phi}_{i}-Z_{\delta / 2} \sqrt{\operatorname{var}(\hat{\phi})}<\phi_{0}<\hat{\phi}_{i}+Z_{\delta / 2} \sqrt{\operatorname{var}(\hat{\phi})}\right)
$$

where $I(\cdot)$ is an indicator function and $\sqrt{\operatorname{var}(\hat{\phi})}$ is the standard error of the estimate $\phi_{i}$. The coverage probability computes the proportion of times the confidence interval contains the true value of the parameter $\phi_{0}$.

Table 3: Monte Carlo simulation results for average bias of the MLE.

\begin{tabular}{c|cccc}
\hline Parameter & $N$ & Average Bias $(\alpha)$ & Average Bias $(\theta)$ & Average Bias $(\lambda)$ \\
\hline & & & & \\
$\alpha=1.0$ & 50 & 0.2970 & 0.0314 & 0.0994 \\
$\theta=0.3$ & 75 & 0.1357 & 0.0293 & 0.0724 \\
$\lambda=0.5$ & 100 & 0.1364 & 0.0082 & 0.0362 \\
& 200 & 0.0507 & 0.0068 & 0.0157 \\
\hline & & & & \\
$\alpha=1.5$ & 50 & 0.4154 & 0.0341 & 0.1622 \\
$\theta=0.4$ & 75 & 0.2859 & 0.0141 & 0.0740 \\
$\lambda=0.6$ & 100 & 0.1714 & 0.0140 & 0.0540 \\
& 200 & 0.0721 & 0.0074 & 0.0242 \\
\hline & & & & 0.2115 \\
$\alpha=2.0$ & 50 & 0.5309 & 0.0403 & 0.0883 \\
$\theta=0.5$ & 75 & 0.2740 & 0.0087 & 0.0319 \\
$\lambda=0.8$ & 100 & 0.2340 & -0.0114 & 0.0513 \\
\hline
\end{tabular}

Table 4: Monte Carlo simulation results for mean square error (MSE) of the MLE.

\begin{tabular}{l|cccc}
\hline Parameter & $N$ & $M S E(\alpha)$ & $M S E(\theta)$ & $M S E(\lambda)$ \\
\hline & & & & \\
$\alpha=1.0$ & 50 & 0.6165 & 0.0727 & 0.2892 \\
$\theta=0.3$ & 75 & 0.2463 & 0.0422 & 0.1285 \\
$\lambda=0.5$ & 100 & 0.2105 & 0.0398 & 0.0916 \\
& 200 & 0.0598 & 0.0146 & 0.0271 \\
\hline
\end{tabular}




\begin{tabular}{l|clll}
\hline$\alpha=1.5$ & 50 & 1.5398 & 0.0995 & 0.4345 \\
$\theta=0.4$ & 75 & 0.8736 & 0.0511 & 0.1777 \\
$\lambda=0.6$ & 100 & 0.3915 & 0.0392 & 0.3917 \\
& 200 & 0.1208 & 0.0197 & 0.0477 \\
\hline & & & & \\
$\alpha=2.0$ & 50 & 3.4213 & 0.0975 & 0.6521 \\
$\theta=0.5$ & 75 & 0.9752 & 0.0568 & 0.2400 \\
$\lambda=0.8$ & 100 & 0.5449 & 0.0408 & 0.1602 \\
& 200 & 0.2143 & 0.0260 & 0.1023 \\
\hline
\end{tabular}

Table 5: Monte Carlo simulation results for coverage probability of the $95 \%$ CIs of the MLE.

\begin{tabular}{l|cccc}
\hline Parameter & $n$ & $C P(\alpha)$ & $C P(\theta)$ & $C P(\lambda)$ \\
\hline & & & & \\
$\theta=1.0$ & 50 & 0.8200 & 0.8133 & 0.8333 \\
$\theta=0.3$ & 75 & 0.8800 & 0.8933 & 0.8933 \\
$\lambda=0.5$ & 100 & 0.9200 & 0.8867 & 0.8967 \\
& 200 & 0.9300 & 0.9400 & 0.9467 \\
\hline & & & & \\
$\alpha=1.5$ & 50 & 0.8667 & 0.8000 & 0.8167 \\
$\theta=0.4$ & 75 & 0.9200 & 0.8967 & 0.8867 \\
$\lambda=0.6$ & 100 & 0.9433 & 0.9233 & 0.9033 \\
& 200 & 0.9500 & 0.9200 & 0.9133 \\
\hline & & & & \\
$\alpha=2.0$ & 50 & 0.9167 & 0.8900 & 0.8700 \\
$\theta=0.5$ & 75 & 0.9567 & 0.9133 & 0.8900 \\
$\lambda=0.8$ & 100 & 0.9700 & 0.9067 & 0.8800 \\
& 200 & 0.9300 & 0.9167 & 0.9233 \\
\hline
\end{tabular}

Tables 3, 4 and 5 present the Monte Carlo simulation results for the average bias, mean square error and coverage probability of the $95 \%$ confidence interval of the parameter estimates of the TLWD at different choice of parameter values. Clearly from Table 3, we observe that the parameter $\alpha$ is positively biased, parameter $\lambda$ is positively biased, while parameter $\theta$ could either be negatively or positively biased. Also from Table 4 , as the sample size $n$ increases, the values of the mean square error of the parameter estimates decreases (tends to zero). Finally, from Table 5, we observe that the coverage probabilities of the CIs are quite close to the nominal level of $95 \%$. 


\section{Application of the Proposed Distribution}

In this section, we fit the proposed distribution to a real data set alongside with some well-known lifetime distributions from the Topp-Leone Generated family of distributions with density functions given by;

(i) Topp-Leone Inverse Weibull Distribution (TLIWD)

$$
f(x)=\frac{2 \alpha \theta \lambda}{x^{\lambda+1}} e^{-\theta / x^{\lambda}}\left(1-e^{-\theta / x^{\lambda}}\right)\left[1-\left(1-e^{-\theta / x^{\lambda}}\right)^{2}\right]^{\alpha-1} ;
$$

(ii) Topp-Leone Bur XII Distribution (TLBXIID)

$$
f(x)=2 \alpha \theta \lambda x^{\theta-1}\left(1+x^{\theta}\right)^{-(2 \alpha+1)}\left[1-\left(1+x^{\theta}\right)^{-2 \alpha}\right]^{\lambda-1} ;
$$

(iii) Topp-Leone Exponential Distribution (TLED)

$$
f(x)=2 \alpha \lambda e^{-2 \lambda x}\left(1-e^{-2 \lambda x}\right)^{\alpha-1} ;
$$

(iv) Topp-Leone Linear Exponential Distribution (TLLED)

$$
f(x)=2 \alpha(\theta+\lambda x) e^{-2\left(\theta x+\frac{\lambda x^{2}}{2}\right)}\left[1-e^{-2\left(\theta x+\frac{\lambda x^{2}}{2}\right)}\right]^{\alpha-1} ;
$$

(v) Topp-Leone Nadarajah-Haghighi Distribution (TLNHD)

$$
f(x)=2 \alpha \theta \lambda(1+\theta x)^{\lambda-1} e^{2\left(1-(1+\theta x)^{\lambda}\right)}\left[1-e^{2\left(1-(1+\theta x)^{\lambda}\right)}\right]^{\alpha-1} .
$$

The dataset consists of the tensile strength, measured in GPa, of 69 carbon fibers tested under tension at gauge lengths of $20 \mathrm{~mm}$ reported in [7]. Table 6 presents the dataset.

Table 6: Tensile strength, measured in GPa, of 69 carbon fibers.

\begin{tabular}{lllllll}
\hline 1.312 & 1.314 & 1.479 & 1.552 & 1.700 & 1.803 & 1.861 \\
1.865 & 1.944 & 1.958 & 1.966 & 1.997 & 2.006 & 2.021 \\
2.027 & 2.055 & 2.063 & 2.098 & 2.14 & 2.179 & 2.224 \\
2.240 & 2.253 & 2.270 & 2.272 & 2.274 & 2.301 & 2.301 \\
2.359 & 2.382 & 2.382 & 2.426 & 2.434 & 2.435 & 2.478 \\
2.490 & 2.511 & 2.514 & 2.535 & 2.554 & 2.566 & 2.57 \\
2.586 & 2.629 & 2.633 & 2.642 & 2.648 & 2.684 & 2.697 \\
2.726 & 2.770 & 2.773 & 2.800 & 2.809 & 2.818 & 2.821 \\
2.848 & 2.88 & 2.954 & 3.012 & 3.067 & 3.084 & 3.090 \\
3.096 & 3.128 & 3.233 & 3.433 & 3.585 & 3.585 & \\
\hline
\end{tabular}


The comparison criteria considered in this paper includes, the estimates of the parameters of the distribution, $\log -l i k$, Akaike Information Criterion $A I C=2 k-$ $2 \log (L)]$, Kolmogorov-Smirnov Statistic $(K-S)$ and Anderson Darling test statistic $\left(A^{*}\right)$ and Crammer-von Mises test statistic $\left(W^{*}\right)$. Where, $k$ is the number of estimated parameters and $L$ is the value of the log-likelihood function evaluated at the parameter estimates.

Table 7: Summary statistics for the tensile strength of 69 carbon fibers dataset.

\begin{tabular}{llllccc}
\hline Models & Estimates & Log-Lik & AIC & $\begin{array}{c}\text { K-S } \\
(p \text {-value })\end{array}$ & $\begin{array}{c}W^{*} \\
(p \text {-value })\end{array}$ & $\begin{array}{c}A^{*} \\
(p \text {-value })\end{array}$
\end{tabular}

\begin{tabular}{|c|c|c|c|c|c|c|}
\hline TLWD & $\begin{aligned} \alpha & =3.8590 \\
\theta & =0.0194 \\
\lambda & =2.0312\end{aligned}$ & -48.8598 & 103.7195 & $\begin{array}{c}0.0395 \\
(0.9999)\end{array}$ & $\begin{array}{c}0.0154 \\
(0.9996)\end{array}$ & $\begin{array}{r}0.1435 \\
(0.9990)\end{array}$ \\
\hline TLBXIID & $\begin{array}{c}\alpha=1.4510 \\
\theta=1.8205 \\
\lambda=110.9177\end{array}$ & -59.9967 & 125.9933 & $\begin{array}{c}0.1216 \\
(0.2591)\end{array}$ & $\begin{array}{c}0.3164 \\
(0.1215)\end{array}$ & $\begin{array}{r}2.1117 \\
(0.0799)\end{array}$ \\
\hline TLIWD & $\begin{array}{c}\alpha=0.5469 \\
\theta=34.8899 \\
\lambda=3.4115\end{array}$ & -58.0304 & 122.0608 & $\begin{array}{c}0.1176 \\
(0.2960)\end{array}$ & $\begin{array}{c}0.2617 \\
(0.1741)\end{array}$ & $\begin{array}{c}1.7344 \\
(0.1294)\end{array}$ \\
\hline TLED & $\begin{array}{c}\alpha=88.1467 \\
\lambda=1.0186\end{array}$ & -54.6201 & 113.2403 & $\begin{array}{c}0.0950 \\
(0.5617)\end{array}$ & $\begin{array}{c}0.1601 \\
(0.3608)\end{array}$ & $\begin{array}{c}1.1210 \\
(0.2994)\end{array}$ \\
\hline TLLED & $\begin{array}{c}\alpha=6.9393 \\
\theta=-0.0826 \\
\lambda=0.4742\end{array}$ & -50.5881 & 107.1762 & $\begin{array}{c}0.0660 \\
(0.9240)\end{array}$ & $\begin{array}{c}0.0627 \\
(0.7985)\end{array}$ & $\begin{array}{r}0.4406 \\
(0.8072)\end{array}$ \\
\hline TLNHD & $\begin{array}{c}\alpha=21.4659 \\
\theta=0.0243 \\
\lambda=17.6504\end{array}$ & -50.4692 & 106.9383 & $\begin{array}{c}0.0652 \\
(0.9311)\end{array}$ & $\begin{array}{c}0.0604 \\
(0.8129)\end{array}$ & $\begin{array}{c}0.4235 \\
(0.8246)\end{array}$ \\
\hline
\end{tabular}

\section{Discussion of Result}

The superiority of a model over another can be characterized by the model having the maximized loglikelihood and the least value in terms of AIC, $K-S$ Statistic, Anderson 
Darling test statistic $\left(A^{*}\right)$ and Crammer-von Mises test statistic $\left(W^{*}\right)$. Table 7 clearly reveal that the proposed distribution (TPGLD) having the maximized loglikelihood and the least values in terms of $A I C, K-S$ Statistic, Anderson Darling test statistic $\left(A^{*}\right)$ and Crammer-von Mises test statistic $\left(W^{*}\right)$, demonstrates superiority over the Topp Leone Inverse Weibull Distribution (TLIWD), Topp Leone Bur XII Distribution (TLBXIID), Topp Leone Exponential Distribution (TLED), Topp Leone Linear Exponential Distribution (TLLED) and Topp Leone Nadarajah-Haghighi Distribution (TLNHD) in modeling the lifetime dataset under study. This claim was further supported by graphical illustration of the density and cumulative distribution fit of the distributions for the real lifetime dataset as displayed in Figure 4.

Density fit for the Data Set

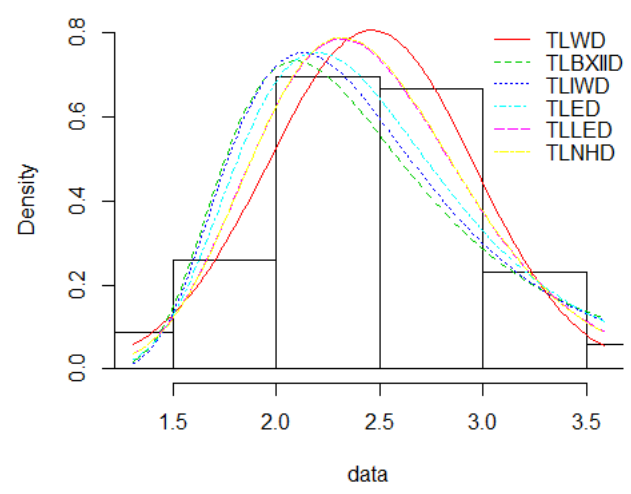

Cumulative distribution fit for the Data Set

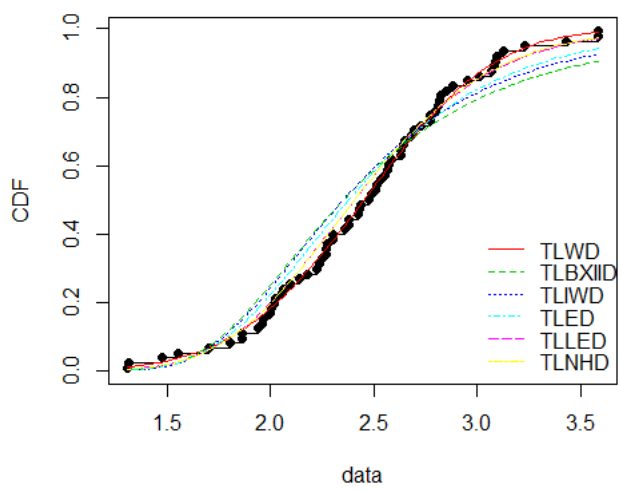

Figure 4: Density and cumulative distribution fit of the distributions for the dataset.

\section{Conclusion}

In this paper, we proposed a new member of the Topp-Leone generated family of distributions called the Topp-Leone Weibull Distribution (TLWD). The mathematical properties of the proposed distribution such as; the density function, cumulative distribution function, survival function, hazard rate function, moments and related measure, moment generating function, quantile function, Renyi entropy and the distribution of ordered statistics were derived. The method of maximum likelihood estimation was used in estimating the parameters of the proposed distribution.

Finally, an application of the proposed distribution to a real lifetime data set alongside with the Topp-Leone Bur XII distribution, Topp-Leone Inverse Weibull distribution,Topp-Leone Exponential distribution, Topp-Leone Linear Exponential distribution, and Topp-Leone Nadarajah-Haghighi distribution, reveals that the proposed 
Topp-Leone Weibull distribution, demonstrates superiority and offers more flexibility in modeling the lifetime data set under study.

\section{References}

[1] S. Abbas, S. A. Taqi, F. Mustafa, M. Murtaza, and M. Q. Shahbaz, Topp-Leone inverse Weibull distribution: theory and application, European Journal of Pure and Applied Mathematics 10(5) (2017), 1005-1022.

[2] A. Al-Shomrani, O. Arif, A. Shawky, S. Hanif and M. Q. Shahbaz, Topp-Leone family of distributions: some properties and application, Pakistan Journal Statistics and Operation Research 12(3) (2016), 443-451. https://doi.org/10.18187/pjsor.v12i3.1458

[3] G. R. Aryal, E. M. Ortega, G. G. Hamedani and H. M. Yousof, The Topp-Leone generated Weibull distribution: regression model, characterizations and applications, International Journal of Statistics and Probability 6(1) (2017), 126-141. https://doi.org/10.5539/ijsp.v6n1p126

[4] S. Aryuyuen, A Topp-Leone generator of exponentiated power Lindley distribution and its application, Applied Mathematical Sciences 12(12) (2018), 567-579. https://doi.org/10.12988/ams.2018.8454

[5] W. Bodhisuwan, The Topp-Leone Gumbel distribution, 12th International Conference on Mathematics, Statistics, and their Applications (ICMSA), Banda Aceh, Indonesia, 2016, pp. 93-98. https://doi.org/10.1109/ICMSA.2016.7954316

[6] N. Ekhosuehi, F. C. Opone and F. Odobaire, A new generalized two parameter Lindley distribution, Journal of Data Science 16(3) (2018), 549-566. https://doi.org/10.6339/JDS.201807_16(3).0006

[7] M. Ghitany, D. Al-Mutairi, N. Balakrishnan and I. Al-Enezi, Power Lindley distribution and associated inference, Computational Statistics and Data Analysis 64 (2013), 20-33. https://doi.org/10.1016/j.csda.2013.02.026

[8] R. D. Gupta and D. Kundu, Generalized exponential distribution, Australian and New Zealand Journal of Statistics 41 (1999), 173-188.

https://doi.org/10.1111/1467-842X.00072

[9] F. C. Opone, and B. N. Iwerumor, A new Marshall-Olkin extended family of distributions with bounded support, Gazi University Journal of Science 34(3) (2021), 899-914. https://doi.org/10.35378/gujs.721816

[10] F. C. Opone, N. Ekhosuehi and S. E. Omosigho, Topp-Leone power Lindley distribution (TLPLD): its properties and application, Sankhyā A: The Indian Journal of Statistics (2020). https://doi.org/10.1007/s13171-020-00209-0 
[11] S. Nadarajah, and S. Kotz, Moments of some J-shaped distributions, Journal of Applied Statistics 30 (2003), 311-317. https://doi.org/10.1080/0266476022000030084

[12] A. Rényi, On measure of entropy and information, Proceedings of the $4^{\text {th }}$ Berkeley Symposium on Mathematical Statistics and Probability 1, University of California Press, Berkeley, 1961, pp. 547-561.

[13] Y. Sangsanit and W. Bodhisuwan, The Topp-Leone generator of distributions: properties and inferences, Songklanakarin Journal of Science and Technology 38 (2016), 537-548.

[14] C. W. Topp and F. C. Leone, A family of J-shaped frequency functions, Journal of the American Statistical Association 50 (1955), 209-219.

https://doi.org/10.1080/01621459.1955.10501259

This is an open access article distributed under the terms of the Creative Commons Attribution License (http://creativecommons.org/licenses/by/4.0/), which permits unrestricted, use, distribution and reproduction in any medium, or format for any purpose, even commercially provided the work is properly cited. 